RUNNING HEAD: Social-emotional devaluation by inhibition

Response inhibition negatively impacts social-emotional evaluations of specific individuals.

Rachel L. Driscoll, Elizabeth M. Clancy, \& Mark J. Fenske

Department of Psychology, University of Guelph, Ontario Canada

Words in abstract: 145

Words in main text body: 5071

Number of references: 67

\title{
Correspondence:
}

Mark J. Fenske, PhD

Department of Psychology

University of Guelph

Guelph, Ontario N1G 2W1

Email: mfenske@uoguelph.ca

Tel: 519-824-4120 x56411

Acknowledgement: This work was supported by the Natural Science and Engineering Research Council of Canada, the Canada Foundation for Innovation, and the Ontario Ministry of Research and Innovation. 


\begin{abstract}
Social-emotional evaluations of unfamiliar people are negatively impacted by ignoring or withholding motor-responses from images that depict them; an effect attributed to the propensity of inhibition to affectively devalue associated stimuli. Prior findings suggest that the socialemotional consequences of inhibition may be mediated by category-level representations that impact all members of a corresponding group. Here we assess whether social devaluation by inhibition also operates on item-level representations of specific individuals. Participants memorized individual identities of a group of fellow students before completing a Go/No-go response-inhibition task designed to associate item-level representations of each previouslymemorized person with inhibition (No-go trials) or no inhibition (Go trials). Social identities associated with inhibition were consistently rated as less trustworthy in subsequent evaluations than those associated with Go trials that were not inhibited. This suggests that the socialemotional consequences of inhibition can be mediated by item-level stimulus representations in memory.
\end{abstract}

Keywords: response inhibition, affective value, social evaluation, trustworthiness, Go/No-go task 


\section{Response inhibition negatively impacts social-emotional evaluations of specific individuals.}

Our understanding of the cognitive processes involved in forming social-emotional impressions of others has been critically informed by studies showing the influence of sociallyrelevant perceptual information, such as that conveyed by an individual's face (e.g., for reviews see Todorov, Olivola, Dotsch \& Mende-Siedlecki, 2015 and Zebrowitz, 2017) or body (e.g., de Gelder et al., 2010; Greven, Downing \& Ramsey, 2019; Musher-Eizenman \& Carels, 2009; Naumann, Vazire, Rentfrow, \& Gosling, 2009), how they are dressed (e.g., Gillath, Bahns, Ge, \& Crandall, 2012; Howlett, Pine, Orakçıŏlu \& Fletcher, 2013; Smith, Liss, Erchull, Kelly, Adragna \& Baines, 2018), or whether they have visible body modifications such as piercings or tattoos (e.g., Broussard \& Harton, 2018; Fung et al, 2019; Westerfield, Stafford, Speroni \& Daniel, 2012). And while there is still much work to be done to identify and elucidate the impact of such external stimulus-linked influences, it is becoming clear that there are also important, yet less obvious, sources of internally-generated affect that can affect our evaluations and the behavioural choices we make. Originating from mental operations, such as those involved in cognitive control, these affective signals provide important feedback related to ongoing performance (Shackman et al., 2011; Shenhav, Botvinivk \& Cohen, 2013). But they also alter our evaluations of associated stimuli (Chetverikov, 2014; Fritz \& Dreisbach, 2013) and the choices we make (Dignath \& Eder, 2015), and thereby have important implications for our understanding of the determinants of human behaviour. For example, task-related factors that affect the fluency or relative ease of cognitive processing during encounters with images of individuals' faces can impact subsequent social evaluations about those individuals (e.g., Winkielman, Olszanowski, \& Gola, 2015; Owen, Halberstadt, Carr, \& Winkielman, 2016). Here we focus on the social-emotional impact of cognitive inhibition - a type of control mechanism 
critically involved in stopping or overriding cognitive/response-related processes - to better understand the type of the representations that mediate this internally-generated influence on our evaluations of others.

Several studies over the last 15 years have established that simply ignoring or withholding motor-responses from face or whole-body images of unfamiliar people can negatively impact subsequent social evaluations of those people, including relatively lower ratings of their trustworthiness (Doallo et al., 2012; Fenske et al., 2005; Frischen et al., 2012; Goolsby, Shapiro, \& Raymond, 2009; Goolsby, Shapiro, Silvert et al., 2009; Kiss et al., 2008; Raymond, Fenske \& Westoby, 2005), likeability (Martiny-Huenger, Gollwitzer \& Oettingen, 2014), attractiveness (Ferrey, Frischen \& Fenske, 2012; Driscoll, Quinn de Launay \& Fenske, 2018), and their ability to garner sympathy (Dickert \& Slovic, 2013). It is clear that inhibitory mechanisms are critically involved both in ignoring distracting information and stopping unwanted actions (for recent reviews see Chelazzi, Marini, Pascucci, \& Turatto, 2019; Duque, Greenhouse, Labruna \& Ivry, 2017; Gaspelin \& Luck, 2018; Noonan, Crittenden, Jensen, \& Stokes, 2018). This has led to the hypothesis — supported by converging cognitive-behavioural (e.g., Frischen et al., 2012), computational modeling (Fragopanagos et al., 2009), neuroimaging (De Vito, Al-Aidroos \& Fenske, 2017; Doallo et al., 2012; Kiss et al., 2008) and psychophysiological (Clancy, Fiacconi \& Fenske, 2019) evidence- that inhibition elicits negative affect or otherwise alters the coding of stimulus-value for associated items (for reviews see Fenske \& Raymond, 2006; Raymond, 2009; Gollwitzer, Martiny-Huenger, \& Oettingen, 2014). This is important in the social domain because eliciting negative affect in others makes people susceptible to social exclusion (e.g., Driscoll, Barclay \& Fenske, 2017). Indeed, we have shown that prior inhibition associated with images of previously-unfamiliar people negatively 
biases evaluative choices away from those individuals (Fenske et al., 2005) and both reduces behavioural approach toward associated social stimuli (Ferrey et al., 2012; Driscoll et al., 2018) and increases behavioural avoidance of them (Driscoll et al., 2018).

The outstanding question we address here is whether the social-emotional consequences of response inhibition can operate on item-level representations of specific individuals, or are mediated instead solely by category-level representations common to all members of the corresponding group. Prior research investigating the impact of inhibition on social-emotional evaluations has so-far only ever utilized attentional- or response-inhibition tasks (e.g., attention: visual search; response: Go/No-go) that require category-level discrimination of the to-beignored/no-response items from the targets of attention/response. Inhibition and the corresponding social devaluation in these studies may have therefore been triggered and associated with category-level representations of the to-be-ignored/no-response individuals according to their membership in a particular socially-relevant group (male/female: Frischen et al., 2012; asian/cacausian: Doallo et al., 2012; Kiss et al., 2008; in-group/out-group: MartinyHuenger et al, 2014; light-haired/dark-haired: Driscoll et al., 2018; Ferrey et al., 2012) or a shared perceptual feature (e.g., colour of a semi-transparent overlay appearing over each face stimulus: red/green, Fenske et al., 2005; blue/yellow, Kiss et al., 2007; Goolsby, Shapiro \& Raymond, 2009; Goolsby, Shapiro, Silvert, et al., 2009). And this may explain why the socialemotional consequences of attention- and response-related inhibition have so far been found to not only impact evaluations of the specific items encountered in the inhibition-based tasks (Driscoll et al., 2018; Fenske et al., 2005; Ferrey et al., 2012; Kiss et al., 2008; Martiny-Huenger et al, 2014), but to also generalize to impact affective responses to other previously-unseen stimuli that belong to the same category or that have the same defining feature as a previously- 
inhibited item (Driscoll et al., 2018; Ferrey et al., 2012; Goolsby et al., 2009; Goolsby, Shapiro, Silvert, et al., 2009).

There is a long history of research that suggests that the formation of impressions that drive social behaviour is, at least at first, primarily dominated by category-level information (e.g., Allport, 1954; Devine, 1989; Fiske \& Neuberg, 1990). Indeed, the influence of such category-level stereotypes can continue to alter social-emotional reactions to a member of a group, even when there is clear information about that specific person that would otherwise lead to a much different reaction (e.g., Eagly et al., 2002; Heilman, Wallen, Fuchs, \& Tamkins, 2004). Such evidence of the relatively greater salience of category-level influences on social judgements makes it unclear whether inhibition and the corresponding social devaluation can also be associated with item-level representations of specific individuals. However, prior proposals about the functional significance of the link between inhibition and aversive response (e.g., Frischen et al., 2012; Raymond et al., 2003; Veling, Holland \& van Knippenberg, 2008) posit that devaluation-by-inhibition effects reflect cooperation of the two main systems of prioritization in the human brain (i.e., emotion and cognitive control) to ensure that specific items previously appearing as distracting or otherwise-inappropriate stimuli can be effectively avoided in the future. And inhibition has been observed to have item-level effects on affective evaluations of specific non-social stimuli, such as logographic symbols (Martiny-Huenger et al., 2014), meaningless shapes (De Vito et al., 2017), and abstract art-like images (Clancy et al., 2019). This suggests that inhibition may also have item-level effects on social-emotional evaluations of specific individuals.

\section{Current Study}

To test whether the social-emotional consequences of inhibition can be mediated by item- 
level representations, we modified a Go/No-go response-inhibition task so that successful performance requires an item-level discrimination regarding the specific person whose face was shown on a given Go/No-go trial. Specifically, participants were shown a stimulus display containing a face and a name, and were asked to judge whether the name belonged to the person depicted in the image. The ability to make this judgement depended on participants first learning the identity of each face stimulus in a social-identity learning task such that they could correctly recall the majority of the names associated with the specific faces. Once the social identities had been learned, the corresponding faces were then presented individually in the Go/No-go task: half of the faces were each shown with their correct name; half were shown with an incorrect name. Participants prepared to press a 'Match' key on trials in which they judged that the name matched the identity of the face (Match condition), and prepared to press a 'No-match' key on trials in which they judged that the name did not match the identity of the face (No-Match condition). Whether participants actually executed their prepared response (Go) or withheld from responding (No-go) was determined by a subsequent change in the colour of a central fixation cross. This Go/No-go task therefore required participants to process and represent a specific social identity at the time of inhibition. A social-emotional evaluation task was then used to assess the trustworthiness of the individuals depicted by each of the face stimuli. If inhibition and the social devaluation that results from it can be associated with item-level representations of specific individuals, then faces encountered on No-go trials should subsequently receive more negative ratings than faces encountered on Go trials. If devaluation-by-inhibition in the social domain is instead mediated only by category-level representations, then ratings of prior No-go faces and prior Go faces should not differ because of the absence of any category-level distinction between the faces used in the two conditions. To anticipate our results, the specific 
faces previously appearing on No-go trials were indeed consistently rated as significantly less trustworthy than those previously appearing on Go trials.

\section{Methods}

The methods used in our experiment were a modified version of those used by Kiss et al. (2008). All of the materials and procedures used in this experiment were approved by the Research Ethics Board at the University of Guelph (REB protocol \#13JN002)

\section{Participants}

A proper test of our hypothesis that the social-emotional consequences of inhibition can be mediated by item-level representations requires that we only consider data from participants who first demonstrate that they have the item-level representations of specific individuals in memory. The specific individuals of interest here are those whose face/name identities were memorized in the Social-identity Learning task before being presented as stimuli in the Go/Nogo and Social-emotional Evaluation tasks. Thus, only participants who were able to reach an initial learning criteria (i.e., correctly recall at least $75 \%$ of the specific individuals) went on to be tested in the Go/No-go and Social-emotional Evaluation tasks. Accordingly, when estimating the required size of our sample, we did not aim for a certain grand-total number of participants, but instead estimated the required number of participants who were able to complete the entire experiment. We therefore recruited — in exchange for course credit - a grand-total of 161 participants (age range: 18-30; sex: 140 female, 21 male) from the University of Guelph undergraduate research participant pool. Of these, 84 participants reached or exceeded the initial learning criteria and then completed the Go/No-go and Social-emotional Evaluation tasks. This resulted in final sample size $(\mathrm{N}=84)$ that was in line with a calculation using $\mathrm{G}^{*}$ Power (Faul, Erdfelder, Lang, \& Buchner, 2007), which indicated that 80 participants would be required to 
detect a same-sized social-emotional effect of response inhibition as that $(d=.82)$ observed by Ferrey et al. (2012, Exp. 2) with a power of 0.95 at an alpha of .05 (the same sample-size calculation was reported by Driscoll et al., 2018). All of the participants provided informed consent and had normal or corrected-to-normal vision.

\section{Stimuli and apparatus}

Stimuli were 54 digital colour photographs of real faces, each paired with a realistic name. Each face stimulus was an image of a University of Guelph student — frontal-view, direct gaze, neutral expression, and visible hair and neck — subtending approximately $10.1^{\circ} \mathrm{x} 12.2^{\circ}$ visual angle at a viewing distance of approximately $60 \mathrm{~cm}$. These stimuli were collected during a prior study in which a different, unique set of University of Guelph undergraduate students provided consent to have their photograph taken and used as part of a future experiment. Half of the images were female faces and half were male faces; together, they reflected the range of racial and ethnic diversity present within the local student population (see Figure 1). The specific name paired with each face was selected from a list of the most popular baby names in the USA in the1990's (https://www.ssa.gov/cgi-bin/popularnames.cgi). Chosen names were two syllables long and, due to their popularity, were considered to be highly familiar and easily processed by the university-age participants. A ' + ' symbol that subtended approximately $0.90^{\circ} \mathrm{x} 0.90^{\circ}$ visual angle was used as a central fixation cross.

Stimulus presentation and behavioral response collection were controlled by E-Prime 2.0 software (Psychology Software Tools, Pittsburgh, PA, USA) running on an Intel Core2Duo computer with a $50.8 \mathrm{~cm}$ LCD monitor (resolution: 1680 x 1050 pixels) and standard keyboard in a private testing room with standard office lighting. All stimuli were presented on a white background. 


\section{Design and Procedure}

The study included three main phases: a Social-identity Learning task, a Go/No-go response-inhibition task, and a Social-emotional Evaluation task (see Figure 1). Instructions were provided prior to each task. The experimenter remained in the testing room throughout the experimental session.

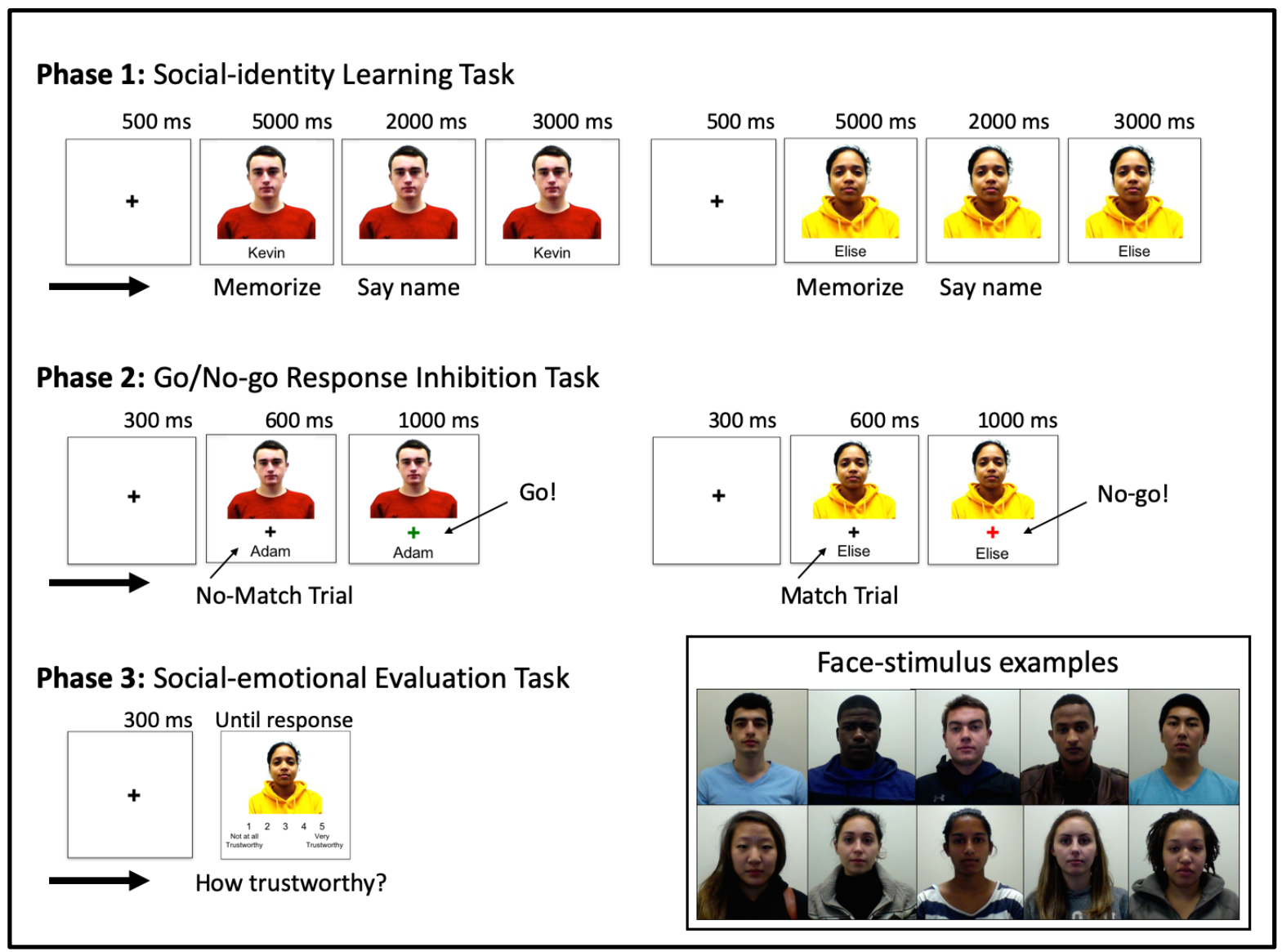

Figure 1. Examples of face-stimuli and sequence of events for each phase of the experiment. Phase 1 required participants to memorize the face-name pair for each social identity until they could correctly recall the name belonging to each face for at least $75 \%$ of the people. Phase 2 was a Go/No-go task based on specific social identities. Participants were asked to assess and get ready to indicate whether the name presented below fixation belonged to the face presented above fixation. The appearance of a Go or No-go signal (fixation turned red vs. green) indicated whether to execute the prepared response (Go) or withhold from responding (No-go). In Phase 3, participants rated the trustworthiness of the individuals depicted by each face image. Socialemotional evaluations were made for each of the people whose identities had been associated with inhibition (prior No-go), not associated with inhibition (prior Go), or had been memorized in Phase 1 but not encountered in the Phase 2 Go/No-go task (Baseline). 
Social-identity Learning. During the learning task, participants were asked to memorize the 54 face-name pairs. The face and name that belonged to each unique social identity was first presented together at display center for $5000 \mathrm{~ms}$. The name was then removed from the display. This served as a prompt for the participant to say the name aloud while continuing to fixate on the face, which remained visible throughout the 2000 ms naming-response window. The experimenter recorded all oral naming responses. The name would then reappear below the face and remain visible for another $3000 \mathrm{~ms}$, which served to confirm the identity of the individual depicted in the face image. A $500 \mathrm{~ms}$ fixation cross separated each of these initial face-name presentations. The order of each of the 54 face-name presentations was randomized for this and each subsequent set of face-name presentations in the Social-identity Learning task.

After the initial face-name presentations and naming responses, participants were shown each face and asked to recall and say aloud the name that belonged to it. The face appeared alone during this $4000 \mathrm{~ms}$ name recall window. Regardless of whether the participant provided a correct response, the name would then appear below the face for $1000 \mathrm{~ms}$ before being removed. This prompted the participant to again say the name aloud while continuing to fixate on the face, which remained visible throughout this $2000 \mathrm{~ms}$ naming-response window. Finally, the facename pair was presented again for $1000 \mathrm{~ms}$. Each round of these recall-and-learn trials therefore provided the opportunity to assess each participant's ability to remember the names that belonged to each of the 54 faces, as well as for participants to better learn these face-name pairings.

At the end of each round of 54 recall-and-learn trials, the experimenter calculated whether the participant had been able to correctly recall the names of at least $75 \%$ of the faces. If the participant reached this learning criteria, they immediately moved on to the Go/No-go and 
Social-emotional Evaluation tasks. If not, they completed another round of recall and learn trials. Participants unable to reach the learning criteria after six rounds of recall-and-learn trials were informed that they had reached the end of their experimental session, debriefed, and thanked for their participation.

Go/No-go. Our modified Go/No-go response-inhibition procedure required participants to process and represent one of the specific social identities they had just learned during each Go/No-go trial. Forty of the 54 face-name pairs were used during experimental Go/No-go trials, with another 4 used in the practice trials. The remaining face-name pairs were not presented in the Go/No-go task, but were instead reserved for use in the Social-emotional Evaluation task to obtain baseline ratings of faces whose identities had been memorized, but were not associated with either response inhibition nor a motor-response. Although the vast majority of prior studies using Go/No-go tasks to investigate the affective consequences of response inhibition used ratings of stimuli associated with Go trials as the main benchmark against which to assess the level of devaluation of stimuli associated with No-go trials, Veling et al. (2008) and Chen, Veling, Dijksterhuis \& Holland (2016) have shown that No-go items are typically also rated more negatively than a baseline comprised of ratings of previously unseen novel items. Thus, we opted to include a similar baseline condition in our study, as well as the standard Go-condition benchmark.

To ensure that participants were processing and representing the identity of the specific person whose face was shown in a given Go or No-go trial, we asked participants to make an item-level discrimination about the face-name pair shown in a stimulus display. Specifically, after an initial $300 \mathrm{~ms}$ central fixation cross, participants were shown a face with a name below it, and were asked to judge during the initial $600 \mathrm{~ms}$ of exposure whether the name belonged to 
the person depicted in the image. The faces on half of the Go/No-go trials were each shown with their correct name, and on the remaining half of trials were shown with an incorrect name. The incorrect names were other options selected from the same list of popular names as used when selecting the original correct names. Participants prepared to press a 'Match' key on trials in which they judged that the name matched the identity of the face (Match condition), and prepared to press a 'No-match' key on trials in which they judged that the name did not match the identity of the face (No-Match condition). Whether participants actually executed their prepared response (Go) or withheld from responding (No-go) was determined by a subsequent change $-600 \mathrm{~ms}$ after the onset of the face-name display — in the colour of the central fixation cross (e.g., green = Go; red = No-go: cue-colour assignment counter-balanced across participants). This Go/No-go cue remained visible with the face-name pair for the remaining $1000 \mathrm{~ms}$ of the trial. On Go trials, participants pressed the Match key or No-match key during this period using their left or right index finger (hand/response assignment counterbalanced across participants).

The assignment of the specific social identities to each of the different conditions of our Go/No-go task were counterbalanced across participants, such that each face was used equally often with its correct name as a Match/Go item and a Match/No-go item, with a different name as a No-Match/Go item and a No-Match/No-go item, and reserved for later use as a Baseline item in the Social-emotional Evaluation task. Participants began the Go/No-go task with a practice block of 16 trials, which could be repeated until the participant indicated they were ready to move on to the experimental blocks. Each of the four experimental blocks contained 10 trials. The order of trials was randomized within each experimental block. 
Social-emotional Evaluation. The Social-emotional Evaluation task required participants to view each of the faces whose identities they had memorized (excluding the four faces used in practice trials) and evaluate their trustworthiness. On each trial, participants were asked "How trustworthy is this person?", with a $300 \mathrm{~ms}$ fixation cross followed by the presentation of a face at display center. Each face appeared with a 1 (not at all trustworthy) to 5 (very trustworthy) scale below it. Both the face and rating scale remained visible until a response was made using the number keys at the top-left of the keyboard. Each of the five rating blocks contained 10 trials. The assignment of faces to the trials within the blocks was randomized for each participant. Participants were instructed to base their ratings on their initial reaction upon seeing each face and to use the full rating scale across trials.

Debrief: Familiarity Rating and Final Recall. Because the face stimuli we used were photographs of other University of Guelph students, we were concerned that some of our participants may recognize the faces as belonging to someone they already know. Thus, after the main experimental tasks, we asked participants to indicate whether they had any prior affiliation with any of the people whose faces were shown in the study. Specifically, participants were shown each face and asked "How well do you know this person in the real-world?" Each face appeared with a 1 (not at all) to 5 (very well) scale below it. This allowed us to identify and exclude from data analysis any trials containing a previously-familiar face to address the possibility of confounds between the affective consequences of our experimental manipulations and any influences that prior real-life experience may have had on participants' social-emotional evaluations. This process also made clear the need to exclude all data from two participants because they knew or recognized multiple people whose face images were used in the study. 
Finally, participants were shown each face for $4000 \mathrm{~ms}$ and asked to say aloud the name of the person depicted in the image. Each of these final-recall trials was separated by a $300 \mathrm{~ms}$ fixation cross. This final-recall task was used as another verification that each participant could recall at least $75 \%$ of the social identities used as stimuli in the experiment.

\section{Results}

Our main measure of interest was the trustworthiness ratings obtained in the Socialemotional Evaluation task of people whose social identities were associated with inhibition (prior No-go trials) compared to those not associated with inhibition (prior Go trials, Baseline). However, because errors are known to elicit their own negative affective consequences (e.g., Chetverikov, 2014), ratings of the identities from the $5.89 \%$ of Go/No-go trials in which an error was made were excluded from our analyses. We also excluded all data from two participants because of low accuracy on No-go trials ( $>2.5$ SD below the mean), because of evidence that poor No-go performance can reflect impairments in the ability to exert inhibition (Aron and Poldrack, 2005). Results are presented in Figure 2 and reported below for the remaining 80 participants (mean age 18.61 years, $\mathrm{SD}=1.31 ; 70$ females).

A 2 (Response-status: Go vs. No-go) x 2 (Face-name: Match vs. No-Match) repeatedmeasures ANOVA revealed that the trustworthiness ratings of people whose identities were associated with inhibition on No-go trials $\left(M_{N o-g o}=3.25, S D=.44\right)$ were significantly lower than those associated with Go trials $\left(M_{G o}=3.37, S D=.38\right)$ [Response-status main effect: $F(1,79)=$ $\left.11.65, p=.001 \eta^{2}=.129\right]$. This result replicates the typical finding of affective devaluation of stimuli associated with No-go trials when compared to the standard Go-condition benchmark, and is therefore consistent with the hypothesis that the social-emotional consequences of inhibition can be mediated by item-level representations. Moreover, the magnitude of this effect 
did not differ across Match and No-Match trials, [Response-status x Face-name interaction: $F(1$, $\left.79)=.70, p=.70 \eta^{2}=.002\right]$. Although the average trustworthiness ratings for people whose identities were associated with inhibition on No-go trials was also nominally lower than the Baseline ratings of people whose identities were previously-memorized but not encountered in the Go/No-go task $\left(M_{\text {Baseline }}=3.27, S D=.47\right)$, a planned-comparison revealed that this difference was not significant, $t(79)=.27, p=.79$.

Although not central to the question of whether the social-emotional consequences of inhibition can be mediated by item-level representations, it is noteworthy that social-emotional evaluations were significantly higher for people whose face appeared with the correct name on Go/No-go trials $\left(M_{\text {Match }}=3.39, S D=.040\right)$ than for those whose face appeared with a different name $\left(M_{\text {No-match }}=3.24, S D=.044\right)$ [Face-name main effect: $\left.F(1,79)=8.60, p=.004 \eta^{2}=.098\right]$.

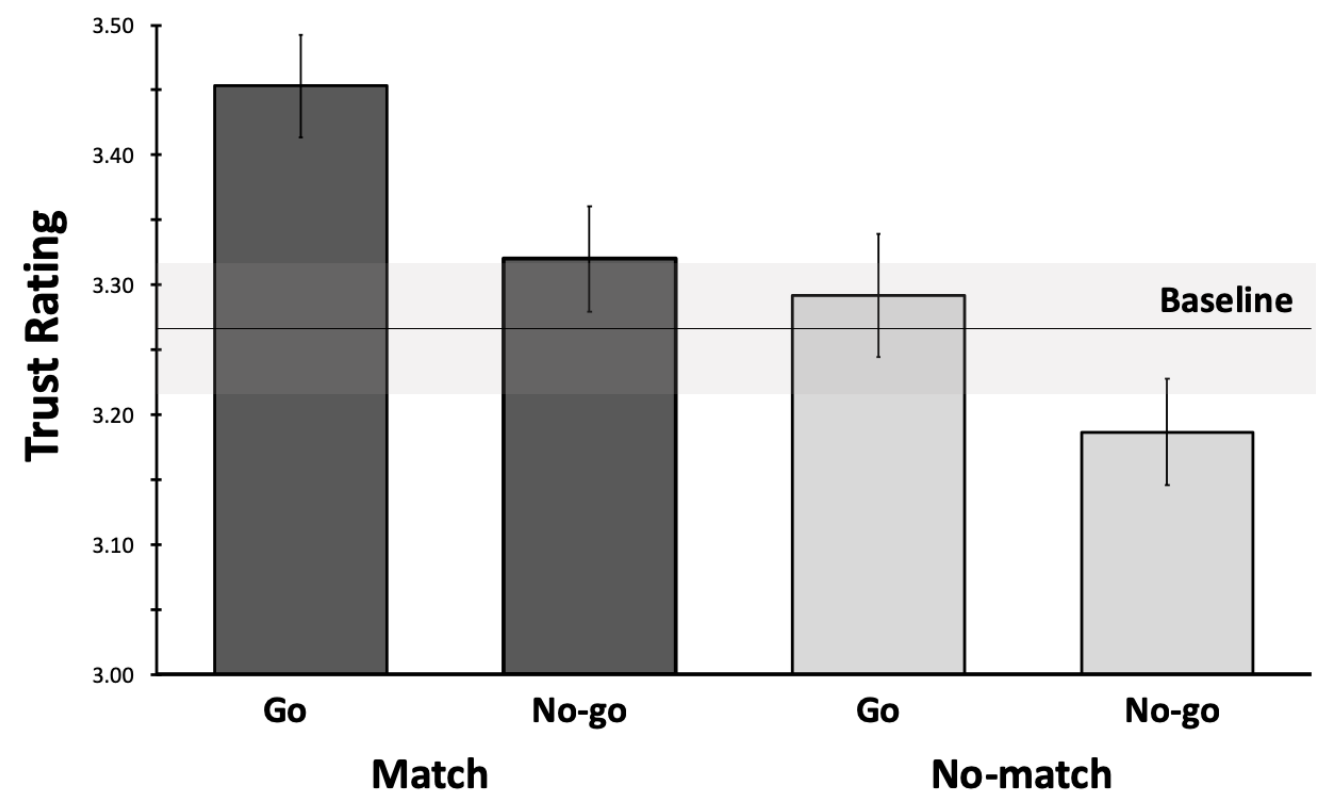

Figure 2. Mean social-emotional evaluations for previously-memorized identities from each experimental condition. Evaluation scores ranged from 1 to 5, with larger values representing more positive ratings of trustworthiness. Error bars represent within-subject standard errors of the means calculated using the Cousineau (2005) method with a Morey (2008) correction. 


\section{Discussion}

Ignoring or withholding motor-responses from face or whole-body images of unfamiliar people can negatively impact subsequent social evaluations of those people, an effect attributed to the propensity of inhibition to affectively devalue associated stimuli (Dickert \& Slovic, 2013; Doallo et al., 2012; Driscoll et al., 2018; Fenske et al., 2005; Ferrey et al., 2012; Frischen et al., 2012; Goolsby et al., 2009; Goolsby, Shapiro, Silvert et al., 2009; Kiss et al., 2008; MartinyHuenger et al., 2014; Raymond et al., 2005). However, because prior studies have so-far only ever utilized attentional- or response-inhibition tasks (e.g., attention: visual search; response: Go/No-go) that require category-level discrimination of the to-be-ignored/no-response items from the targets of attention/response, it has remained unclear whether inhibition and the corresponding social devaluation is solely triggered and associated with category-level representations of the to-be-ignored/no-response individuals according to their membership in a particular socially-relevant group or a shared perceptual feature, or can also be mediated by itemlevel representations in memory. We addressed this by modifying a Go/No-go responseinhibition task to require participants to process and represent a specific social identity at the time of inhibition, and found that the subsequent trustworthiness ratings of people whose identities were associated with inhibition on No-go trials were significantly lower than those not associated with inhibition on Go trials. If social devaluation by inhibition were mediated solely by category-level representations, then evaluations of prior No-go individuals and prior Go individuals should not differ because of the absence of any category-level distinction between the social identities used in our experimental conditions. Our results are therefore consistent instead with the hypothesis that the social-emotional consequences of inhibition can be mediated by item-level representations in memory. These new findings in the social domain extend prior 
observations of inhibition-related devaluation of specific stimuli in the non-social domain (e.g., Clancy et al., 2019; De Vito et al., 2017; Martiny-Huenger et al., 2014).

Our results converge with growing evidence that the changes in stimulus value observed following tasks that involve attentional (e.g., De Vito et al., 2017; Fenske, Raymond \& Kunar, 2004; Fenske et al., 2005; Goolsby et al., 2009, Kiss et al., 2007; Martiny-Huenger et al., 2014; Raymond et al., 2003; 2005), response (e.g., Clancy et al., 2019; Driscoll et al., 2018; Frischen et al., 2012; Ferrey et al., 2012; Kiss et al., 2008), and memory-related inhibition (De Vito \& Fenske, 2017; 2018; De Vito et al., 2018; Vivas et al., 2016) may be due to the affective consequences of inhibition per se. However, some caution is warranted when considering the extent to which our results support this interpretation because the level of social devaluation for people whose identities were associated with inhibition on No-go trials was only significant when using the standard Go-condition benchmark; it was not significant when using Baseline ratings of people whose identities were previously-memorized but not encountered in the Go/Nogo task. And while the vast majority of prior studies have only used the Go-condition benchmark to assess levels of stimulus devaluation by inhibition, the use of Baseline ratings that have not been influenced by Go/No-go task performance has the added benefit of allowing the negative effects of response inhibition during No-go trials to be distinguished from any positive effects of active responding during Go trials. With this in mind, it is noteworthy that evidence that active responding enhances stimulus evaluations has so-far been limited (e.g., observed by Schonberg et al., 2014, but not in any of the three experiments of Veling et al., 2008), and appears to be depend on stringent conditions that require rapid Go-trial responses (see Chen et al., 2016). With $1600 \mathrm{~ms}$ to prepare and make each Go response, our experimental procedure clearly did not include such conditions. Nevertheless, having an appropriate baseline condition to make direct 
comparisons of effects on ratings linked to a No-go condition versus those linked to a Go condition would be helpful because, to the best of our knowledge, such a comparison has never been done for affective evaluations in the social domain.

Unfortunately, the usefulness of the additional Baseline condition in our experiment may be limited by the unanticipated effect of whether each face on Go/No-go trials appeared with the correct name (Match) or not (No-match). We used these Match and No-match conditions as a technique to ensure participants would be actively processing and maintaining item-level representations of a specific previously-memorized social identity of the person whose face was shown on each Go/No-go trial. It was effective in its intended purpose to the extent that we observed significant differences in subsequent ratings of the specific individuals appearing in prior No-go and Go trials. However, it is clear that this technique also inadvertently introduced meaningful differences in processing fluency (Match $>$ No-match) — a factor known to elicit positive affect (Winkielman, Schwarz, Fazendeiro, \& Reber, 2003) and enhance social evaluations (e.g., Winkielman, Olszanowski, \& Gola, 2015), and/or differences in cognitive conflict (No-match $>$ Match) — a factor known to elicit negative affect (Dreisbach \& Fischer, 2012) and decrease affective evaluations (e.g., Damen, Strick, Taris, \& Aarts, 2018). An assessment of how quickly participants made correct responses on Go trials revealed that average response times on Match trials were significantly faster than on No-match trials $(M=607 \mathrm{~ms}$ vs. $652 \mathrm{~ms} ; t(79)=5.19, p<0.0001)$, providing evidence of more fluent processing of social identities on Match trials and/or more conflicted processing on Non-match trials. The face-name matching effect we observed clearly depends on item-level representations of specific individuals and may therefore be of interest on its own as another example of how affective signals originating from mental operations alter our evaluations of specific stimuli. However, it 
complicates the usefulness of our Baseline condition because the social evaluations of individuals from each of our experimental conditions are affected, not just by the presence or absence of inhibition, but also by the potential positive effect of fluency and/or the negative effect of cognitive conflict. Without a Baseline condition that is properly matched for level of fluency/conflict, it is impossible to unequivocally distinguish the negative effects of response inhibition during No-go trials from any potential effects of active responding during Go trials. Although our addition of the memorized-only Baseline condition did not work out as planned, our results nevertheless clearly replicate the typical finding of affective devaluation of stimuli associated with No-go trials when compared to the standard Go-condition benchmark. The task demands of prior studies meant that inhibition and the corresponding social devaluation may have been triggered and associated with category-level representations of the to-beignored/no-response individuals according to their membership in a particular socially-relevant group (Doallo et al., 2012; Driscoll et al., 2018; Ferrey et al., 2012; Frischen et al., 2012; Kiss et al., 2008; Martiny-Huenger et al, 2014) or a shared perceptual feature (Fenske et al., 2005; Goolby et al., 2009; Goolsby, Shapiro, Silvert et al., 2009; Kiss et al., 2007). In contrast, all of the social stimuli in our experiment belonged to the same group as the participants-fellow students - with successful performance in the response-inhibition task depending on memory for the specific person whose face was shown on a given Go/No-go trial. Thus, the novel finding from our experiment is that inhibition and the social devaluation that results from it is not mediated solely by category-level representations common to all members of the corresponding group, but can also be associated with item-level representations of specific individuals. 


\section{References}

Allport, G.W. (1954). The nature of prejudice. Reading, MA: Addison-Wesley.

Aron, A.R. \& Poldrack, R.A. (2005). The cognitive neuroscience of response inhibition:

relevance for genetic research in attention-deficit/hyperactivity disorder. Biological Psychiatry, 57(11), 1285-92.

Broussard, K.A., \& Harton, H.C. (2018). Tattoo or taboo? Tattoo stigma and negative attitudes toward tattooed individuals. The Journal of Social Psychology, 158(5), 521-540.

Chelazzi L., Marini F., Pascucci D., Turatto M. (2019). Getting rid of visual distractors: The why, when, how and where. Current Opinion in Psychology, 29, 135-147.

Chen, Z., Veling, H., Dijksterhuis, A., \& Holland, R.W. (2016). How does not responding to appetitive stimuli cause devaluation: Evaluative conditioning or response inhibition? Journal of Experimental Psychology: General, 145(12), 1687-1701.

Chetverikov, A. (2014). Warmth of familiarity and chill of error: Affective consequences of recognition decisions. Cognition \& Emotion, 28(3), 385-415.

Clancy, E.M., Fiacconi, C. \& Fenske, M.J. (2019). Response inhibition immediately elicits negative affect and devalues associated stimuli: Evidence from facial electromyography. Progress in Brain Research, 247, 169-191.

Damen, T.G.E., Strick, M., Taris, T.W., Aarts, H. (2018) When conflict influences liking: The case of the Stroop task. PLOS ONE 13(7): e0199700.

de Gelder, B., van den Stock, J., Meeren, H.K.M., Sinke, C.B.A., Kret, M.E., $\&$ Tamietto, M. (2010). Standing up for the body. Recent progress in uncovering the networks involved in the perception of bodies and bodily expressions. Neuroscience \& Biobehavioral Reviews, 34, 513-527. 
De Vito, D., \& Fenske, M.J. (2017). Suppressing memories of words and familiar objects results in their affective devaluation: Evidence from Think/No-think tasks. Cognition, 162, 1-11.

De Vito, D., \& Fenske, M.J. (2018). Affective evidence that inhibition is involved in separating accessory representations from active representations in visual working memory. Visual Cognition, 26(8), 583-600.

De Vito, D., Al-Aidroos, N., \& Fenske, M.J. (2017). Neural evidence that inhibition is linked to the affective devaluation of distractors that match the contents of working memory. Neuropsychologia, 99, 259-269.

De Vito, D., Ferrey, A.E., Fenske, M.J., \& Al-Aidroos, N. (2018). Cognitive-behavioral and electrophysiological evidence of the affective consequences of ignoring stimulus representations in working memory. Cognitive, Affective, \& Behavioral Neuroscience, 18(3), 460-475.

Devine, P. G. (1989). Stereotypes and prejudice: Their automatic and controlled components. Journal of Personality and Social Psychology, 56(1), 5-18.

Dickert, S., \& Slovic, P. (2013). Attentional mechanisms in the generation of sympathy. Judgement and Decision Making, 4, 297-306.

Dignath, D., \& Eder, A. B. (2015). Stimulus conflict triggers behavioral avoidance. Cognitive, Affective, \& Behavioral Neuroscience, 15(4), 822-836.

Doallo, S., Raymond, J.E., Shapiro, K.L., Kiss, M., Eimer, M., \& Nobre, A.C. (2011). Response inhibition results in the emotional devaluation of faces: neural correlates as revealed by fMRI. Social Cognitive and Affective Neuroscience, 7(6), 649-659.

Dreisbach, G., \& Fischer, R. (2012). Conflicts as aversive signals. Brain and Cognition, 78, 9498. 
Driscoll, R.L., Barclay, P., \& Fenske, M.J. (2016). To be spurned no more: the affective and behavioral consequences of social and non-social rejection. Psychonomic Bulletin and Review: $1-8$.

Driscoll, R.L., De Launay, K.Q., \& Fenske, M.J. (2018). Less approach, more avoidance: Response inhibition has motivational consequences for sexual stimuli that reflect changes in affective value not a lingering global brake on behavior. Psychonomic Bulletin \& Review, 25(1), 463-471.

Duque J., Greenhouse I., Labruna L., Ivry R.B. (2017) Physiological markers of motor inhibition during human behavior. Trends in Neuroscience, 40, 219 -236.

Eagly, A.H., \& Karau, S.J. (2002). Role congruity theory of prejudice toward female leaders. Psychological Review, 109(3), 573-598.

Faul, F., Erdfelder, E., Lang, A.G., \& Buchner, A. (2007). G* Power 3: A flexible statistical power analysis program for the social, behavioral, and biomedical sciences. Behavior Research Methods, 39(2), 175-191.

Fenske, M.J., \& Raymond, J.E. (2006). Affective influences of selective attention. Current Directions in Psychological Science, 15(6), 312-316.

Fenske, M.J., Raymond, J.E., \& Kunar, M.A. (2004). The affective consequences of visual attention in preview search. Psychonomic Bulletin \& Review, 11, 1034-1040.

Fenske, M.J., Raymond, J.E., Kessler, K., Westoby, N., \& Tipper, S.P. (2005). Attentional inhibition has social-emotional consequences for unfamiliar faces. Psychological Science, 16, $753-758$. 
Ferrey, A.E., Frischen, A., \& Fenske, M.J. (2012). Hot or not: response inhibition reduces the hedonic value and motivational incentive of sexual stimuli. Frontiers in Psychology, 3, 575.

Fiske, S.T., \& Neuberg, S. L. (1990). A continuum of impression formation, from categorybased to individuating processes: Influences of information and motivation on attention and interpretation. In Advances in Experimental Social Psychology (Vol. 23, pp. 1-74). Academic Press.

Fragopanagos, N., Cristescu, T., Goolsby, B.A., Kiss, M., Eimer, M., Nobre, A.C., ... \& Taylor, J.G. (2009). Modelling distractor devaluation (DD) and its neurophysiological correlates. Neuropsychologia, 47(12), 2354-2366.

Frischen, A., Ferrey, A.E., Burt, D.H.R., Pistchik, M., \& Fenske, M.J. (2012). The affective consequences of cognitive inhibition: Devaluation or neutralization? Journal of Experimental Psychology: Human Perception and Performance, 38(1), 169-179.

Fritz, J., and Dreisbach, G. (2013). Conflicts as aversive signals: Conflict priming increases negative judgments for neutral stimuli. Cognitive, Affective, \& Behavioral Neuroscience, $13,311-317$.

Fung, N., Ishii, M., Huynh, P., Juarez, M., Bater, K., Darrach, H., ... \& Ishii, L. (2019). Stretched Earlobe Piercings Negatively Impact Casual Observer Perceptions. Facial Plastic Surgery, 35(03), 299-305.

Gaspelin N. \& Luck S.J. (2018). The role of inhibition in avoiding distraction by salient stimuli. Trends in Cognitive Sciences, 22(1), 79-92.

Gillath, O., Bahns, A.J., Ge, F., \& Crandall, C.S. (2012). Shoes as a source of first impressions. Journal of Research in Personality, 46(4), 423-430. 
Gollwitzer, P.M., Martiny-Huenger, T., \& Oettingen, G. (2014). Affective consequences of intentional action control. In Advances in Motivation Science (Vol. 1, pp. 49-83). Elsevier.

Goolsby, B.A., Shapiro, K.L., \& Raymond, J.E. (2009). Distractor devaluation requires visual working memory. Psychonomic Bulletin \& Review, 16(1), 133-138.

Goolsby, B.A., Shapiro, K.L., Silvert, L., Kiss, M., Fragopanagos, N., Taylor, J.G., ... \& Raymond, J.E. (2009). Feature-based inhibition underlies the affective consequences of attention. Visual Cognition, 17(4), 500-530.

Greven, I.M., Downing, P.E., \& Ramsey, R. (2019). Neural networks supporting social evaluation of bodies based on body shape. Social Neuroscience, 14(3), 328-344.

Heilman, M.E., Wallen, A.S., Fuchs, D., \& Tamkins, M.M. (2004). Penalties for success: reactions to women who succeed at male gender-typed tasks. Journal of Applied Psychology, 89(3), 416-427.

Howlett, N., Pine, K., Orakçığlu, I., \& Fletcher, B. (2013). The influence of clothing on first impressions: Rapid and positive responses to minor changes in male attire. Journal of Fashion Marketing and Management: An International Journal, 17(1), 38-48.

Greven I.M, Downing P.E., \& Ramsey R. (2019) Neural networks supporting social evaluation of bodies based on body shape. Social Neuroscience, 14:3, 328-344.

Kiss, M., Goolsby, B. A., Raymond, J.E., Shapiro, K.L., Silvert, L., Nobre, A.C., ... \& Eimer, M. (2007). Efficient attentional selection predicts distractor devaluation: Event-related potential evidence for a direct link between attention and emotion. Journal of Cognitive Neuroscience, 19(8), 1316-1322. 
Kiss, M., Raymond, J.E., Westoby, N., Nobre, A., \& Eimer, M. (2008). Response inhibition is linked to emotional devaluation: behavioural and electrophysiological evidence. Frontiers in Human Neuroscience, 2: 13.

Martiny-Huenger, T., Gollwitzer, P.M., \& Oettingen, G. (2014). Selective attention to in-and out-group members systematically influences intergroup bias. Social Psychological and Personality Science, 5(8), 936-943.

Musher-Eizenman, D., \& Carels, R.A. (2009). The impact of target weight and gender on perceptions of likeability, personality attributes, and functional impairment. Obesity Facts, 2, 311-317.

Naumann, L. P., Vazire, S., Rentfrow, P.J., \& Gosling, S.D. (2009). Personality judgments based on physical appearance. Personality and Social Psychology Bulletin, 35, 1661-1671.

Noonan M.P., Crittenden B.M., Jensen O., Stokes M.G. (2018) Selective inhibition of distracting input. Behavioural Brain Research. 355, 36-47.

Owen, H.E., Halberstadt, J., Carr, E.W., \& Winkielman, P. (2016). Johnny Depp, reconsidered: How category-relative processing fluency determines the appeal of gender ambiguity. PloS one, 11(2), e0146328.

Raymond, J.E. (2009). Interactions of attention, emotion and motivation. Progress in Brain Research, 176, 293-308.

Raymond, J.E., Fenske, M.J., \& Tavassoli, N.T. (2003). Selective attention determines emotional responses to novel visual stimuli. Psychological Science, 14: 537-542.

Raymond, J.E., Fenske, M. J., \& Westoby, N. (2005). Emotional devaluation of distracting patterns and faces: A consequence of attentional inhibition during visual search? Journal of Experimental Psychology: Human Perception and Performance, 31(6), 1404-1415. 
Shackman, A.J., Salomons, T.V., Slagter, H.A., Fox, A.S., Winter, J.J., Davidson, R.J. (2011). The integration of negative affect, pain and cognitive control in the cingulate cortex. Nature Reviews Neuroscience, 12: 154-167.

Shenhav, A., Botvinick, M.M., \& Cohen, J.D. (2013). The expected value of control: an integrative theory of anterior cingulate cortex function. Neuron, 79: 217-240.

Schonberg, T., Bakkour, A., Hover, A.M., Mumford, J.A., Nagar, L., Perez, J., \& Poldrack, R. A.. (2014). Changing value through cued approach: an automatic mechanism of behavior change. Nature Neuroscience, 17, 625-630.

Smith, J.K., Liss, M., Erchull, M.J., Kelly, C.M., Adragna, K., \& Baines, K. (2018). The relationship between sexualized appearance and perceptions of women's competence and electability. Sex Roles, 79(11-12), 671-682.

Todorov, A., Olivola, C.Y., Dotsch, R., \& Mende-Siedlecki, P. (2015). Social attributions from faces: Determinants, consequences, accuracy, and functional significance. Annual Review of Psychology, 66, 519-545.

Veling, H., Holland, R.W., \& van Knippenberg, A. (2008). When approach motivation and behavioral inhibition collide: Behavior regulation through stimulus devaluation. Journal of Experimental Social Psychology, 44(4), 1013-1019.

Vivas, A.B., Marful, A., Panagiotidou, D., \& Bajo, T. (2016). Instruction to forget lead to emotional devaluation. Cognition, 150, 85-91.

Westerfield, H.V., Stafford, A.B., Speroni, K.G., \& Daniel, M.G. (2012). Patients' perceptions of patient care providers with tattoos and/or body piercings. JONA: The Journal of Nursing Administration, 42(3), 160-164. 
Winkielman, P., Schwarz, N., Fazendeiro, T., \& Reber, R. (2003). The hedonic marking of processing fluency: Implications for evaluative judgment. In J. Musch \& K.

C. Klauer (Eds.), The Psychology of Evaluation: Affective Processes in Cognition and Emotion (pp. 189-217). Mahwah, NJ: Erlbaum.

Winkielman, P., Olszanowski, M., \& Gola, M. (2015). Faces in-between: Evaluations reflect the interplay of facial features and task-dependent fluency. Emotion, 15(2), 232.

Zebrowitz, L.A. (2017). First impressions from faces. Current Directions in Psychological Science, 26(3), 237-242. 